DOI: $10.1590 / 1807-57622013.0446$

\title{
Clínica-escola:
}

\section{apoio institucional inovador às práticas de gestão e atenção na saúde como parte da integração ensino-serviço}

\author{
Olinda Maria de Fátima Lechmann Saldanha(a) \\ Ana Lúcia Bender Pereira(b) \\ Cássia Regina Gotler Medeiros(c) \\ Gisele Dhein ${ }^{(\mathrm{d})}$ \\ Lydia Christmann Espindola Koetz ${ }^{(\mathrm{e})}$ \\ Suzana Feldens Schwertner ${ }^{(f)}$ \\ Ricardo Burg Ceccim ${ }^{(g)}$
}

Saldanha OMFL, Pereira ALB, Medeiros CRG, Dhein G, Koetz LCE, Schwertner SF, et al. Clinic-school: innovative institutional support to the practices of management and health care as part of the teaching-work integration. Interface (Botucatu). 2014; 18 Supl 1:1053-62.

The text presents the implementation of a school-service that proposes health professionals' education in line with the National Curriculum Guidelines. The Regional University Clinic of Education and Health (CURES) involves health courses offered by a University Center located in the region of the Taquari Valley, in the state of Rio Grande do Sul (Southern Brazil), linking the care networks of four cities. This paper reports on the institutional support that is present in the actions of the care network teams. Inclusion in the local and regional context has shown an educational potential for students and teachers, highlighting commitment and co-responsibility in care. The "education narrative" was the methodological approach that was employed to analyze the actions of CURES concerning institutional support to the teams. The inseparability between technique and policy/theory and practice has permeated activities and reflections, resulting in changes in the teaching-learning process in the field of health.

Keywords: Clinic-school. Comprehensive health care. Teaching-work integration. Professional health education. Institutional support.
O texto apresenta a implementação de um serviço-escola que tem como proposta a formação de profissionais da saúde em consonância com as Diretrizes Curriculares Nacionais. A Clínica Universitária Regional de Educação e Saúde (CURES) envolve cursos da área da saúde de um Centro Universitário situado na região do Vale do Taquari/RS, Brasil, articulando as redes de cuidado de quatro municípios. Neste trabalho relata-se o apoio institucional nas ações das equipes da rede de cuidados. A inserção no contexto locorregional tem se mostrado um potencial formativo para estudantes e docentes, ressaltando o compromisso e a corresponsabilização no cuidado. A "narrativa de formação" foi o recurso metodológico utilizado, problematizando as ações da CURES no apoio institucional às equipes. $A$ indissociabilidade entre técnica e política / teoria e prática tem permeado as atividades e reflexões, resultando em modificações no processo ensino-aprendizagem em saúde.

Palavras-chave: Clínica-escola. Atenção integral à saúde. Integração ensinoserviço. Formação profissional em saúde. Apoio institucional.

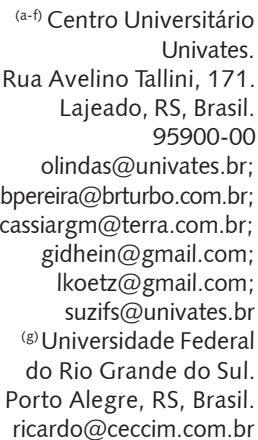




\section{Introdução}

O presente trabalho trata de uma "narrativa de formação" na implementação de um serviço-escola inaugurado em março de 2011. A proposta foi a da construção de uma formação interdisciplinar e multiprofissional de estudantes que envolvesse as competências e habilidades gerais e específicas previstas pelas Diretrizes Curriculares Nacionais (DCN) às profissões da área da saúde e construísse - ao mesmo tempo em que ensinasse - o apoio institucional às práticas de gestão e atenção numa base locorregional, como demandado na "regionalização" prevista pelo Sistema Único de Saúde (SUS).

O "apoio institucional", conforme a Política Nacional de Humanização no SUS', é um dispositivo de intervenção que se propõe às mudanças que o sistema de saúde requer para que se afirme como política pública inclusiva e universal. É proposto como "disparador" na articulação das redes de atenção em saúde e como "conector" dos pontos de rede, produzindo novos territórios, borrando e embaralhando lugares previamente constituídos ${ }^{2,3}$ para desenhar linhas de cuidado e atenção integral.

O serviço-escola, aqui relatado, nomeado por Clínica Universitária Regional de Educação e Saúde (CURES), envolve todos os cursos da área da saúde do Centro Universitário Univates, localizado no município de Lajeado, Região do Vale do Taquari, Estado do Rio Grande do Sul. Foram estabelecidos convênios do Centro Universitário com quatro municípios da região, prevendo-se relações de cooperação regional da instituição formadora com as esferas de gestão pública da saúde e contato com os trabalhadores "das pontas" de rede. Entre as propostas do serviço-escola estava a implementação de ações que promovessem a formação de profissionais comprometidos com as diretrizes constitucionais do SUS e com as diretrizes nacionais aos currículos da área da saúde.

É consenso a opinião de que as diretrizes constitucionais do SUS dificilmente serão implementadas sem um correspondente perfil de formação dos profissionais de saúde ${ }^{4,5,6}$. Várias iniciativas, então, neste sentido estão em curso em todo o país, estimuladas pelas DCN e, também, pelas medidas de apoio do Ministério da Saúde (MS), adquirindo força, sobretudo, a partir do lançamento do AprenderSUS - o SUS e os Cursos de Graduação da Área de Saúde -, política de apoio aos processos de mudança na graduação pela aprendizagem do SUS, do trabalho em equipe e da integralidade 6 . Clínicas-escola, em princípio, não se encaixariam nestas condições, mas estão previstas com obrigatoriedade para alguns cursos, assim como serviços de especialidade seguem formatados para as instituições de ensino e os maiores hospitais-escola permanecem ligados ao sistema de ensino.

Identificando-se com a Reforma Sanitária e com o conceito ampliado de saúde, a política inaugural do AprenderSUS afirmava a perspectiva de uma abordagem que pudesse articular saberes e práticas multiprofissionais e interdisciplinares, orientados pelas bases locais de inserção dos serviços, interrogando-se pela integralidade e resolutividade da atenção, reforçando o debate sobre a universalidade desse direito e seu acesso7. Tal "política" foi sucedida por "ações programáticas" como o Programa de Reorientação da Formação dos Profissionais da Área da Saúde (Pró-Saúde) e o Programa de Educação pelo Trabalho para o Sistema Único de Saúde (PET-Saúde), destinados ao desenvolvimento de cenários de prática da atenção integral e práticas interprofissionais ao longo da graduação.

Verificamos que as mudanças ainda são incipientes, ancoradas em uma cultura hegemônica que mantém as práticas, tanto de ensino como de trabalho em saúde, voltadas a uma proposta anterior à criação do SUS, que persiste no cotidiano dos serviços de saúde e das instituições de ensino. Estas práticas clássicas privilegiam o ensino direcionado à transmissão de conteúdos, tecnicista e descontextualizado das necessidades de saúde da população brasileira. Isto se reflete na não-atenção aos princípios da integralidade, equidade e resolutividade, propostos pela Constituição Brasileira de 1988.

Conforme Pinheiro ${ }^{8}$, o perfil profissional desejado como resultante do Ensino Superior não é apenas o de bons ou excelentes técnicos, mas o de trabalhadores para o SUS, sensibilizados por ações de cuidado que reafirmem a garantia do direito à saúde. Por outro lado, podemos refletir que a forma como os serviços de saúde atuam interfere na formação do estudante, pois este realiza estágios nos serviços e vivencia uma realidade que nem sempre é problematizada pelos docentes, que também estão imersos e são frutos deste contexto. O ensino e o serviço, portanto, participam da formação e da construção do perfil do egresso dos cursos de graduação.

Como destacam Pontes, Silva Junior e Pinheiro ${ }^{4}$, 
existem preconceitos e dificuldades de relação nos serviços de saúde. Na maioria das vezes, os serviços também são fundados no modelo hegemônico de assistência e de formação de profissionais. Suas práticas de saúde reproduzem e incentivam as práticas aprendidas nas universidades e, ao mesmo tempo, criticam suas inadequações, desconsiderando-se como parte dessa formação. (p. 3)

A CURES, como espaço de ensino-aprendizagem-atenção em saúde, foi proposta com várias estratégias de ação, entre elas, a atuação no apoio institucional à rede de cuidados e aos municípios, especialmente abordando o conceito e prática de região de saúde e integralidade da atenção.

Buscamos, com o presente trabalho, analisar o apoio institucional em seu potencial de promover movimentos e mudanças nos serviços, além de instigar a potência formativa destas práticas para docentes e estudantes. $\mathrm{O}$ apoio institucional possibilita às equipes apoiadas a ampliação da sua capacidade de análise, na medida em que mediatiza a reflexão acerca das dificuldades do cotidiano. O fazer e o agir entram na roda, mobilizando a circulação de afetos, de desejos, de interesses e a constituição de vínculos, como parte das estratégias de mudança das condições e da organização do trabalho 9 .

\section{De que serviço-escola estamos falando?}

A CURES é um espaço de atenção e formação interdisciplinar onde docentes e estudantes dos cursos de Educação Física, Enfermagem, Farmácia, Fisioterapia, Nutrição e Psicologia desenvolvem atendimentos individuais, atividades com grupos e oficinas, além do apoio matricial, apoio institucional e discussão de redes, entre outras práticas demandadas pelas equipes e municípios conveniados ou pelos usuários. Uma vez que este serviço não possui profissionais técnicos contratados, os docentes têm a responsabilidade de supervisionar as ações realizadas independentemente da área de formação, o que traz à prática a problematização produzida por diferentes olhares, instigando à reflexão das ações.

Os cursos de graduação da área da saúde em questão foram criados em momentos diferentes, voltados à sua área específica e marcados pelos modelos de produção de saúde hegemônicos no país. Estes modelos são caracterizados pela fragmentação e pela aprendizagem de técnicas e procedimentos centrados no conceito de doença e na intervenção individual, em discrepância com a proposta éticopolítica do SUS, que preconiza a integralidade da atenção, tomando a saúde como resultado das condições de vida e orientada pela participação ativa e efetiva dos usuários ${ }^{10,11}$. Uma clínica e laboratórios de habilidades em clínica estão propostos aos cursos da área da saúde, sem que os mesmos sirvam às habilidades interdisciplinares e ao apoio à gestão do SUS.

A integralidade supõe a inclusão das pessoas numa rede assistencial onde o foco das ações em saúde passa a ser o usuário, e não a fragmentação do cuidado pelo tratamento focal das doenças, numa lógica de horizontalização dos saberes. Para tanto, uma equipe de professores, envolvendo estudantes e estagiários, organizou-se de modo a pensar estratégias coletivas e de desenvolvimento de ações interdisciplinares, com o intuito de criar pontos de contato entre diferentes saberes, capazes de transformar a realidade institucional e dos usuários.

Neste sentido, a CURES integra-se à rede de cuidados em saúde dos municípios conveniados, visando à Educação Permanente em Saúde (EPS) e à regionalização da saúde, numa integração entre Educação Superior, equipes dos serviços, gestão do sistema de saúde e participação dos usuários, valendo-se da orientação política do "Quadrilátero da Formação".

Segundo Ceccim e Feuerwerker 5 , o "quadrilátero da formação" foi cunhado como conceito para representar um novo arranjo de atores relevantes nas definições de estratégias para a educação na saúde, que procura reunir ensino, atenção, gestão e participação em saúde. Seu principal objetivo é dar conta da complexidade, diversidade e amplitude da produção da vida humana, que exige uma formação de pessoal qualificada para a compreensão e a intervenção em estratégias de promoção da saúde como qualidade da vida.

Dessa forma, concomitante aos atendimentos dos usuários, que acontecem a partir das necessidades de saúde - ou outras - identificadas e pactuadas com o usuário, ocorre a qualificação dos trabalhadores 
de saúde e a formação dos estudantes. Contrapondo a lógica formativa das clínicas-escolas específicas dos cursos, a CURES não se limita a um projeto formativo de estudantes voltado às especificidades/particularidades de cada área de conhecimento, e sim a promover a atenção integral no cuidado, com vistas ao desenvolvimento da rede locorregional de atenção à saúde e aperfeiçoamento da gestão do setor.

O apoio à rede pauta-se na crença de que o apoio institucional envolve os atores sociais em suas experiências concretas de relações cotidianas, uma vez que as mudanças não se fazem sem um processo de produção subjetiva, que é sempre coletivo, histórico e condicionado por múltiplos vetores inseparáveis no campo da saúde: sujeitos, processos de trabalho, poder e políticas públicas².

De maneira geral, podemos dizer que o conceito de clínica com o qual a CURES trabalha vai ao encontro daquele proposto pela Saúde Coletiva, que tem como eixo a integralidade da atenção, construída a partir do conceito ampliado de saúde, tendo por base a perspectiva ético-estético-política. Barros $^{11}$ cita a perspectiva ético-estético-política de Félix Guattari: "ético porque potência ativa que surge na imanência das práticas para coordenar a vida e escolher a forma de vivê-la; estético porque criação permanente, subvertendo a pretensa unidade do mundo capitalista; e político porque implica a escolha de modos de mundo que se quer viver" (p. 41).

Entre as propostas da CURES está a promoção da EPS. Embora seja algo novo e desafiador, entendese que este é um caminho necessário/fundamental para que se possa tornar interdisciplinaridade e intersetorialidade práticas possíveis e indispensáveis no trabalho em saúde. Nesta perspectiva, os trabalhadores atuam não como "recursos" administrativos, e sim como "atores sociais", protagonistas da mudança, com capacidade de autoanálise e autogestão, implicando-se, desta forma, no processo de trabalho como "agentes intercessores", com foco na valorização do trabalho em saúde e no acolhimento dos usuários ${ }^{12}$.

Pensar, dialogar, problematizar e estudar as práticas em saúde torna ativa a prática da EPS. O cruzamento de saberes teóricos e práticos, numa atuação interdisciplinar, possibilita, além da aprendizagem efetiva e criativa, capacidade crítica e produtora de sentidos, construindo/produzindo atores ativos das cenas de formação e trabalho. Conforme Ceccim 12, "os saberes formais devem estar implicados com movimentos de autoanálise e autogestão dos coletivos da realidade, pois são os atores do cotidiano que devem ser protagonistas da mudança de realidade desejada pelas práticas educativas" (p. 166).

Diante da necessidade de um novo olhar sobre a saúde, suas práticas e seus atores sociais, a CURES nasce com alguns diferenciais, no intuito de desacomodar docentes, estudantes, profissionais e gestores da saúde a olhar, escutar e cuidar de forma ampliada, constituindo uma nova potência criadora e produtora de vida.

\section{A narrativa de formação como possibilidade metodológica}

A narrativa é um fenômeno antropológico. Narrar é humano, faz parte do processo civilizatório, introduzindo a escrita como aparato tecnológico e colocando o narrador no centro da história. É no século XX que Pineau coloca a escrita de si como arte profissional, como consequência de uma sociedade biográfica, onde o indivíduo assume-se como autor de sua história e responsabiliza-se por ela ${ }^{13}$.

Conforme Scholze ${ }^{14}$, "a produção de narrativas pode ser vista como uma prática de reflexão do sujeito consigo mesmo, com o outro e com o mundo" (p. 89). A autora cita Foucault, afirmando que o sujeito fala, sempre, desde um lugar, não sendo dono de seus atos discursivos. Não é o sujeito monovalente que se expressa, e sim é expresso por todas as narrativas que o constituem. Por sua voz, falam as instituições, os ensinamentos recebidos, os silenciamentos impostos, os discursos permitidos e aqueles estimulados.

O 'quem sou' "é o entrecruzamento das histórias que me narram e me constituem" ${ }^{14}$ (p. 96). As narrativas implicam uma ruptura histórica e um posicionamento político, e falam de um processo e de uma relação: " a finalidade de escrever sobre a própria aprendizagem é justamente a de poder (aprender a) situar-se, deliberadamente, do lado do processo e não do produto, da ação e não da produção, pois se volta para a relação da pessoa com o conhecimento"13 (p. 35). 
Segundo Barbosa, Câmara e Passeggi ${ }^{15}$, a abordagem autobiográfica nos permite problematizar as ações teórico-práticas num desafio de autoavaliação e elaboração de um projeto profissional, numa permanente busca e construção dos sentidos profissionais, sinalizando de onde vim, onde estou e para onde vou.

O foco deste tipo de estudo é o significado que os pesquisadores elaboram e põem em jogo nos discursos, ações e interações, em uma perspectiva onde tanto formas verbais quanto não verbais as produzem. Os "dados" funcionam como "casos-pensamento", não como descritores de uma realidade a ser testada ou representada, não demandando categorização a partir do outro, mas a proposição de eixos, perspectivas ou cenários. O trabalho funciona como um referencial crítico-conceitual embasado numa realidade "experimentada".

A narrativa de formação é relativa ao percurso de um aprender, contemplando o aprendizado como um caminho do pensamento. Tomar o caminho do aprendizado e encontrar sua produção é relatar a inteligência dele decorrente. Não se tratando de uma biografia, a narrativa de formação contempla saberes biográficos ou saberes da experiência. Reunidos esses saberes numa documentação narrativa, possibilitamos o conhecimento de percursos, acumulações e transformações, num registro histórico e no próprio ato narrativo.

\section{Diálogos e movimentos a partir da inserção do serviço-escola nas redes locais}

No presente texto, a narrativa é constituída das experiências de apoio institucional realizadas pela equipe da CURES, na interação com os serviços que integram a rede dos municípios conveniados. Neste sentido, entende-se:

o apoiador não é simplesmente um consultor, que palpita sobre o trabalho e diz das mazelas do grupo; nem tampouco sua ação se resume à assessoria, indicando caminhos a partir de um suposto saber externo que atua sobre o grupo. Sem negar estas especificidades, o apoiador é alguém que penetra no grupo para acioná-lo como dispositivo, apresentando como um 'fora dentro incluído', alguém que atravessa o grupo não para feri-lo, ou para anunciar suas debilidades, para operar junto com o grupo em um processo de transformação na própria grupalidade e nos modos de organizar o trabalho e de ofertar ações e estratégias de saúde. ${ }^{16}$ (p. 14)

Barros $^{17}$ afirma que a produção do conhecimento é uma prática social que só se efetiva quando enfrenta o constituído, criticando-o e transformando-o em outra realidade. A autora refere que, quando as formas de ação perdem sua garantia de neutralidade, constituem-se em instrumentos importantes para efetuar mudanças no plano coletivo e conformar novas políticas de saúde.

É importante destacar que, na CURES, o apoio institucional emerge da problematização que ocorre na construção dos Projetos Terapêuticos Singulares (PTS) entre as suas equipes e as dos serviços dos municípios. Esta problematização evidenciou a fragilidade de integração entre os serviços na constituição de rede de cuidados. Na construção do PTS, os profissionais envolvidos são convidados a compor a equipe de referência na rede de cuidado, identificar necessidades de saúde e construir suas perguntas. O usuário e seus familiares também compõem esta rede, conforme a singularidade do cuidado ${ }^{18}$. O PTS é uma importante variação da discussão de caso clínico, ou seja: um conjunto de propostas de conduta é arrolado pela microequipe do cuidado, identificando-se por onde circula o usuário, o que faz ou poderia fazer a rede de saúde a que pertence, como os gestores locais se envolvem ou poderiam se envolver com o usuário e sua equipe de referência, quais saberes os trabalhadores da rede têm ou deveriam desenvolver sobre a intervenção em situação. Além das contribuições e elevação da capacidade de análise, a roda de conversa estabelecida propõe saberes formativos inéditos/criativos, mais que saber melhor o que é e como atender do ponto de vista clínico.

A partir da definição pela realização das ações com as equipes de cada município, são agendados os encontros com as equipes locais, que são mensais. O primeiro encontro tem como objetivo escutar os profissionais quanto às suas dificuldades, demandas, necessidades e expectativas. A sequência ocorre 
com a problematização das questões apresentadas e a cada encontro é indicada, coletivamente, uma proposta ou temática para o momento seguinte. Dependendo dos movimentos e necessidades do grupo no intervalo entre os encontros, a temática pode ser alterada. Ao final de cada encontro, é feita uma avaliação, por meio da manifestação de todos os participantes.

Pode-se considerar que o maior objetivo e um dos desafios das redes de cuidado em saúde é garantir integralidade, equidade e resolutividade. Nessa experiência narrada, as redes colocam atores diversos a conversar. Por exemplo, historicamente, sabemos que a rede de Educação e a rede de Saúde pouco interagem. Na construção de uma rede de cuidados, esta articulação é fundamental para garantir que as necessidades dos usuários e dos sistemas locais de saúde sejam contempladas. Nas palavras de Inojosa ${ }^{19}$ :

as redes são teias flexíveis e abertas de relacionamentos, mantidas pelo fluxo de compartilhamento de informações, ideias, experiências, ideais, objetivos, esforços, riquezas e necessidades entre os entes que as compõem em determinado momento histórico. (p. 37)

De acordo com Mattos $^{20}$, a rede de assistência tem se constituído na oferta de serviços descompromissados, com as consequências dessa oferta sobre o perfil epidemiológico da população, ordenando o sistema de forma descontextualizada às necessidades e problemas de saúde das pessoas. A rede cuidadora, conforme Silva Junior e Mascarenhas ${ }^{21}$, reconhece a interdependência dos atores e organizações, e estabelece mecanismos de cooperação e coordenação a fim de responder às necessidades de saúde individuais em âmbitos local e regional.

A experiência aqui tematizada vem mostrando quanto é necessária a articulação de "rodas em redes". Encontramos na assistência social, por exemplo, muitas vezes, uma prática ainda centrada no assistencialismo. Na educação, uma preocupação intensa com os resultados homogêneos em sala de aula e com propostas pedagógicas prescritivas direcionadas aos alunos. Na saúde, as dificuldades de sair de um modelo curativista e, em alguns momentos, "moralizante" nas condutas de autocuidado. No momento que se articula a produção de um PTS com vários atores, para compor estratégias de cuidado e gestão, se consegue ressignificar e compor outra lógica de assistência e gerenciamento, maior compromisso e disposição a "fazer falar" as várias instâncias envolvidas na atenção integral à saúde.

Perguntas surgem: como a articulação dos serviços se constituirá numa rede? Podemos desenvolver apoio institucional intersetorial ou somente ao setor da saúde? Estas perguntas nos mostram quanto a formação em saúde ainda se afasta das ações de rede, da integralidade, da interdisciplinaridade, das linhas de cuidado e da locorregião.

$\mathrm{Na}$ CURES, se propõe a constituição de um modelo muito distinto das clínicas-escola existentes na experiência precedente dos cursos da área da saúde e, mesmo, previstas nas DCN de diversos cursos, propondo: apoiar uma organização em rede dos sistemas locais com os quais se referencia; estabelecer um diálogo real com as equipes locais que as fortaleçam e as façam entrar em educação permanente; que sirva de escuta de necessidades de ensino-aprendizagem pelos docentes, estudantes e gestores, tendo em vista, em primeiro lugar, as diretrizes e os princípios do SUS e, na sequência, o artigo comum da área da saúde nas DCN (Art. $4^{\circ}$ ) e parágrafo único do artigo relativo às competências e habilidades específicas $\left(\right.$ Art. $\left.5^{\circ}\right)$.

Conforme Ceccim e Ferla22, a organização da atenção e de uma rede de serviços implica, necessariamente, o conhecimento dos fatores que beneficiam ou prejudicam os estados de saúde e dos recursos existentes para a afirmação da vida, do cuidado de si e das conexões em redes sociais.

Uma das ações que se mantém desde o início do funcionamento da CURES, sistematicamente, é conhecer cada município conveniado. Isto implica diferentes ações, tais como: análise de situação de saúde, levantamento de informações acerca das escolas de cada município, de organizações não governamentais, de projetos sociais, de espaços de lazer, de grupos, oficinas e atividades de produção da saúde ou da cultura. Conhecer estas possibilidades facilita - no apoio institucional - a construção de redes, incentivos à gestão e construção do PTS.

O estranhamento, por parte dos estudantes, de que estas são informações que serão pertinentes ao processo de cuidado, coloca o desafio da busca por estratégias de aproximação entre teorias estudadas e a pertinência em conhecer as necessidades locorregionais de forma contínua e de desconstruir as 
práticas especializadas. Constituir-se apoiador institucional provoca mudanças na formação dos profissionais de saúde, nos docentes e nos estudantes. Este aprendizado, se não for inexistente na maioria dos cursos, não é frequente.

Quando realizadas as reuniões de apoio institucional com os serviços, constata-se que, muitas vezes, é nestes momentos que os trabalhadores dos municípios conversam pela primeira vez entre si. Em alguns casos, consegue-se identificar quanto este apoio institucional produz efeitos na articulação intersetorial: uma Agente Comunitária de Saúde que se aproxima do conselho tutelar para conversar sobre as famílias que ela visita; uma enfermeira que solicita informações à escola de um usuário para pensar as ações de cuidado que vai indicar; um fisioterapeuta que refere o contato com os ambientes de circulação social dos idosos; um farmacêutico que quer participar do grupo de adolescentes, por exemplo.

A cada encontro, novos fluxos vão se estabelecendo na rede, mostrando, como referem Malta e Merhy ${ }^{23}$, que a busca das pessoas pela resposta às suas necessidades é um caminhar nômade, que, geralmente, não conhecemos. As transformações e a construção de redes não acontecem apenas com a implantação das tecnologias duras (ou de referência especializada), nem das tecnologias leve-duras (indicando melhores protocolos ou novos aportes científicos), mas pelas conexões que se pode estabelecer.

O apoio institucional às equipes mostrou-se como um dispositivo para promover a discussão de estratégias de cuidado e reorganização de ações em rede, estimulando mudanças nos processos de trabalho e qualificação da comunicação, tanto entre os diferentes setores e políticas (intersetorialidade) do município como entre estes e a equipe da CURES. Para Campos e Domitti ${ }^{24}$, o apoio procura construir e ativar espaço para a comunicação ativa e para o compartilhamento de conhecimento entre profissionais de referência e apoiadores.

Intercalamos a vinda das equipes municipais à CURES e a ida de docentes e estudantes para os municípios, a fim de conhecermos a estrutura e promovermos a aproximação com outros trabalhadores, em um constante movimento de ensinar-aprender. De acordo com Barros ${ }^{17}$, a problematização não apenas serve "para podermos esboçar novas saídas para os impasses vividos, como, principalmente, para promover outras perguntas, outras entradas" (p. 142).

\section{Algumas considerações de encerramento}

A narrativa de formação, além de reconstruir fatos experienciados, obviamente sob uma ótica local e uma determinada visão de mundo, impregnada de subjetividade, possui o potencial de provocar reflexões e questionamentos, tanto para o próprio narrador quanto para o leitor. A narrativa de formação contempla o rigor da roda de conversa sistemática, saberes biográficos ou da experiência, registro compartilhado e autoria pelos atores sociais da implicação - neste texto, os atores sociais de condução da experiência e sua consultoria.

As DCN dos cursos da área da saúde reforçam a necessidade da inserção dos estudantes na rede de gestão e atenção, para que estes vivenciem a realidade do sistema de saúde, que, na maioria das vezes, não está presente nas clínicas-escola, como as noções de matriciamento, rede locorregional e sistema descentralizado e participativo de saúde. A CURES foi proposta em ruptura com o modelo clássico de clínica-escola, optando-se, em sua criação, pela aprendizagem da interdisciplinaridade entre professores e alunos e na própria instituição de ensino; pelo respeito às diretrizes e princípios do SUS, apoiando municípios e região, não pela oferta de serviço de referência, mas serviço de apoio institucional e educação permanente, não apenas no âmbito da atenção, mas da gestão. Uma clínica-escola que trabalhasse colocando docentes e estudantes em contato com os serviços de saúde (reais/locais), construindo projetos e ações a partir das necessidades levantadas em contexto, operando com a articulação em rede: de cuidados, intersetorial e de gestão.

Conforme Ceccim e Ferla ${ }^{22}$, as relações entre o mercado de trabalho, as estruturas acadêmicas e a prática educativa são constituídas por interfaces de mão dupla, sendo que uma mudança não se constrói plenamente sem a outra. É preciso pensar continuamente a interface formação, produção de subjetividade e trabalho. Para os docentes e estudantes de graduação da área da saúde, as vivências na 
avaliação, planejamento e implementação do apoio institucional aos profissionais da rede e aos municípios têm oportunizado problematizar os fazeres e os saberes, na composição de práticas de pensamento, ação e formação.

Assim como as mudanças na formação, as mudanças no serviço também são complexas, e não basta inserir o estudante nos serviços de saúde se as práticas lá realizadas não forem problematizadas, e outras, mais condizentes com os princípios do SUS, não forem sugeridas e experimentadas. Tais mudanças não se furtam aos afetos, conforme sugerem Ceccim e Ferla ${ }^{22}$.

A inserção do estudante neste contexto se dá como um ator social que constrói, transforma e projeta a sua própria formação, tendo como referência as condições de vida e saúde da população, assim como a organização dos serviços e do sistema de saúde, implicando-se ativamente com a realidade.

Considerando o objeto desta narrativa, verifica-se que o apoio institucional tem produzido movimentos de mudança na rede de cuidados nos municípios. Mudanças estas que são identificadas numa maior comunicação entre equipes e profissionais de diferentes áreas e setores; na implicação do trabalhador com a efetividade da proposta do SUS numa relação promotora de sistemas locais e num processo contínuo de análise, pactuação e educação permanente, considerando as especificidades locorregionais.

Dar forma de texto à experiência é parte da proposta da CURES: criticar, pertencer e ampliar a qualidade da rede locorregional, ao mesmo tempo pedir seu apoio institucional, como feito com o convite a docentes de diversas universidades brasileiras. Vive-se um desenho em movimento: ao mudarem conceitos, mudaram as redes de conversas; ao mudarem as redes de conversas, mudaram os conceitos, mudou o entorno, mudaram as relações.

Em que pese uma aposta na rede de saúde como espaço prioritário e fundamental à aprendizagem dos estudantes, muitas clínicas universitárias sobrevivem ou são abertas, inclusive como modo de atender às DCN ou prioridades do SUS; unidades universitárias são criadas em regiões sem oferta suficiente de serviços para a população local e para a composição de relevantes cenários de aprendizagem dos estudantes; então, a aposta aqui relatada tem mérito ao debate acadêmico e relevância às possíveis construções sociais na integração ensino-serviço na saúde.

\section{Colaboradores}

Olinda Maria de Fátima Lechmann Saldanha, Ana Lúcia Bender Pereira, Cássia Regina Gotler Medeiros, Gisele Dhein, Lydia Christmann Espindola Koetz e Suzana Feldens Schwertner participaram, igualmente, das etapas de elaboração e redação do artigo. Ricardo Burg Ceccim participou da orientação e discussão do artigo e da revisão do texto.

\section{Referências}

1. Ministério da Saúde. Secretaria de Atenção à Saúde. Política Nacional de Humanização. Cadernos HumanizaSUS, v. 2. Atenção Básica - Série B. Textos Básicos de Saúde. Brasília: MS; 2010.

2. Guedes CR, Roza MMR, Barros MEB. O apoio institucional na Política Nacional de Humanização: uma experiência de transformação das práticas de produção de saúde na rede de atenção básica. Cad Saude Colet. 2012; 20(1):93-101. 
3. Vasconcelos MFF, Morschel A. O apoio institucional e a produção de redes: do desassossego dos mapas vigentes na saúde coletiva. Interface (Botucatu). 2009; 13 Supl. 1:729-38.

4. Pontes $A$, Silva Junior AG, Pinheiro R. Ensino da saúde e a rede de cuidados nas experiências de ensino-aprendizagem. In: Pinheiro R, Ceccim RB, Mattos RA, organizadores. Ensinar saúde: a integralidade e o SUS nos cursos de graduação na área da saúde. Rio de Janeiro: Abrasco; 2006. p. 251-73.

5. Ceccim RB, Feuerwerker LCM. O quadrilátero da formação para a área da saúde: ensino, gestão, atenção e controle social. Physis. 2004; 14(1):41-65.

6. Ministério da Saúde. Secretaria de Gestão do Trabalho e da Educação na Saúde. Departamento de Gestão da Educação na Saúde. AprenderSUS: o SUS e os cursos de graduação da área da saúde. Brasília: MS; 2004.

7. Macedo MC, Romano RAT, Henriques RLM, Pinheiro R, Ceccim RB. Cenários de aprendizagem: interseção entre os mundos do trabalho e da formação. In: Pinheiro R, Ceccim RB, Mattos RA, organizadores. Ensinar saúde: a integralidade e o SUS nos cursos de graduação na área da saúde. Rio de Janeiro: Abrasco; 2006. p. 229-50.

8. Pinheiro R. As práticas do cotidiano na relação oferta e demanda nos serviços de saúde: um campo de estudo e construção da integralidade. In: Pinheiro R, Mattos RA, organizadores. Os sentidos da integralidade na atenção e no cuidado à saúde. Rio de Janeiro: Abrasco; 2003. p. 65-112.

9. Barros SCM, Dimenstein M. O apoio institucional como dispositivo de reordenamento dos processos de trabalho na atenção básica. Estud Pesqui Psicol. 2010; 10(1):48-67.

10. Centro Universitário Univates. Resolução no 092 - Reitoria/Univates, de 23 de julho de 2008. Projeto da Clínica Universitária Regional de Educação e Saúde. Lajeado, 2008.

11. Barros MEB. Procurando outros paradigmas para a educação. Educ Soc. 2000; 21(72): $32-42$

12. Ceccim RB. Educação permanente em saúde: desafio ambicioso e necessário. Interface (Botucatu). 2004/2005; 9(16):161-8.

13. Passeggi MC. Memoriais auto-bio-gráficos: a arte profissional de tecer uma figura pública de si. In: Passeggi MC, Barbosa TMN, organizadores. Narrativas de formação e saberes biográficos. Natal: EDUFRN; 2008. p. 27-42.

14. Scholze, L. Narrativas de si e a possibilidade de ressignificação da existência. In: Passeggi M, Barbosa TMN, organizadores. Narrativas de formação e saberes biográficos. Natal: EDUFRN; 2008. p. 89-100.

15. Barbosa TMN, Câmara SCX, Passeggi MC. Memoriais de formação e ensaios autobiográficos tecidos com laços intergeracionais. In: Passeggi MC, Barbosa TMN, organizadores. Memórias, memoriais: pesquisa e formação docente. Natal: EDUFRN; 2008. p. 219-35.

16. Ministério da Saúde. Curso de formação de formadores e apoiadores para a Política Nacional de Humanização da Gestão e da Atenção à Saúde. Projeto de Cooperação Técnica. Brasília: MS; 2006.

17. Barros MEB. Desafios ético-políticos para a formação dos profissionais de saúde. In: Pinheiro R, Ceccim RB, Mattos RA, organizadores. Ensinar saúde: a integralidade e o SUS nos cursos de graduação na área da saúde. Rio de Janeiro: Abrasco; 2006. p. 131-50.

18. Ministério da Saúde. Clínica ampliada, equipe de referência e projeto terapêutico singular. Atenção Básica - Série B. Textos Básicos de Saúde. Brasília: MS; 2008.

19. Inojosa RM. Revisitando as redes. Divulg Saude Debate. 2008; (41):36-46. 
20. Mattos RA. Integralidade e a formulação de políticas específicas de saúde. In: Pinheiro R, Mattos RA, organizadores. Construção da integralidade: cotidiano, saberes e práticas em saúde. Rio de Janeiro: Abrasco; 2003. p. 45-59.

21. Silva Junior AG, Mascarenhas MTM. Avaliação de redes de atenção à saúde: contribuições da integralidade. In: Pinheiro R, Mattos RA, organizadores. Gestão em redes: práticas de avaliação, formação e participação na saúde. Rio de Janeiro: Abrasco; 2006. p. 61-89.

22. Ceccim RB, Ferla AA. Linha de cuidado: a imagem da mandala na gestão em rede de práticas cuidadoras para uma outra educação dos profissionais de saúde. In: Pinheiro R, Mattos RA, organizadores. Gestão em redes: práticas de avaliação, formação e participação na saúde. Rio de Janeiro: Abrasco; 2006. p. 165-84.

23. Malta DC, Merhy E. O percurso da linha do cuidado sob a perspectiva das doenças crônicas não transmissíveis. Interface (Botucatu). 2010; 14(34):593-605.

24. Campos GWS, Domitti AC. Apoio matricial e equipe de referência: uma metodologia para gestão do trabalho interdisciplinar em saúde. Cad Saude Publica. 2007; 23(2):399-407.

Saldanha OMFL, Pereira ALB, Medeiros CRG, Dhein G, Koetz LCE, Schwertner SF, et al. Clínica-escuela: apoyo institucional innovador para las prácticas de gestión y atención en la salud como parte de la integración enseñanza-servicio. Interface (Botucatu). 2014; 18 Supl 1:1053-62.

El texto presenta la implementación de una actividad-escuela, cuya propuesta es la fomación de profesionales de acuerdo con las Directrices Curriculares Nacionales. La Clínica Universitaria Regional de Educación y Salud (CURES) involucra cursos del área de la salud de un Centro Universitario, articulando las redes de cuidado de cuatro municipios brasileños. En este trabajo se hace el relato del apoyo institucional en las acciones de los equipos de la red de cuidados. La inserción en el contexto ha mostrado un potencial formativo para estudiantes y docentes, resaltando el compromiso y la coresponsabilidad en el cuidado. La "narrativa de formación" ha sido el recurso metodológico utilizado, problematizando las acciones de la CURES en el apoyo institucional a los equipos. La indisolubilidad entre técnica y política/teoría y práctica ha traspasado las actividades y las reflexiones.

Palabras clave: Clínica-escuela. Atención integral de la salud. Integración enseñanzaservicio. Formación en salud. Apoyo institucional. 